\title{
Simulation of applied principles of envelope functions for Fabry-Perot spectroscopy of plane wave for single-layer structures
}

\author{
P.S. Kosobutskyy \\ National University "Lviv Polytechnic”, 13, Bandery str., 79646 Lviv, Ukraine \\ E-mail: petkosob@yahoo.com
}

\begin{abstract}
The method to obtain analytical expressions for envelope functions in spectra of normal incidence light reflection and transmission by single-layer structures is proposed.
\end{abstract}

Keywords: Fabry-Perot interferometry, single-layer structure, reflection, transmission, envelope function method.

Manuscript received 11.10.06; accepted for publication 26.03.07; published online 01.06.07.

\section{Introduction}

The principle of multibeam interferometry of light for plane parallel plate developed by Fabry-Perot [1] have been widely used for solving a large number of scientific and applied problems and have been discussed in many textbooks [2-5]. Neveretheless, a number of new regularities in the scope of the problem were recently found. It was shown that Fabry-Perot interferometry is still actual for non-destructive control of optical parameters of micro- and nano-size layers [6-8].

Recently, the so-called method of envelope functions as tangential to contours of amplitude spectra of light reflection and transmission by a single-layer Fabry-Perot structures [10-19] was developed. So, for example, on the basis of the method the approach for determining the variation parameters in thickness and influence of spectral distribution of light and light absorption [9-15] was grounded. The envelope function method was used for grounding the new approach for determination of the instrumental characteristics of interference bands $[16,17]$ and for reconstruction of the phase of a reflected wave by single-layer films $[16,17$, 19]. In other our papers [20-26], we developed the envelope function method for arbitrary ratio between the indices of refraction $n_{1,2,3}$ of three media.

In the paper, authors generalized the basic principles of application of the envelope function method to the data analysis in amplitude-phase spectroscopy of light interference for single-layer structures of Fabry-Perot type (SLSFP) in the case of normal incidence of a beam onto the interfaces for transparent and absorptive structures. A method for obtaining the analytical expression for energetic coefficients $(R, T)_{\max , \min }$ and phase $\phi_{\max , \min }$ is proposed.

\section{General relations}

The SLSFP of a thickness $d$ and of the complex refraction index $\tilde{n}_{2}=n_{2}-i \chi_{2}$ is bounded by the semiinfinite top transparent media with refraction index $n_{1}$ (interface 12) and bottom (transparent or absorbing) media with refraction index $\tilde{n}_{3}$ (interface 23 ). In the absorbing layer, the phase change of wave is equal to $\widetilde{\delta}=\frac{4 \pi d}{\lambda} \tilde{n}_{2}$. It is well known also that taking into account the multibeam interference the complex amplitude values of light reflection $\tilde{r}$ and transmission $\tilde{t}$ by SLSFP are determined from

$$
\tilde{r}=\frac{\tilde{r}_{12}+\tilde{r}_{23} \exp (-i \tilde{\delta})}{1+\tilde{r}_{12} \tilde{r}_{23} \exp (-i \tilde{\delta})} \text { and } \tilde{t}=\frac{\tilde{t}_{12} \tilde{t}_{23} \exp (-i \tilde{\delta} / 2)}{1+\tilde{r}_{12} \tilde{r}_{23} \exp (-i \tilde{\delta})} \text {, }
$$

where $\tilde{r}_{12,23}$ and $\tilde{t}_{12,23}$ are the well-known amplitude Fresnel's coefficients [2] for the single interfaces with subscripts 12 and 23. The resonant dispersion of the media is modelled by one-oscillator function of permittivity [27]

$$
\widetilde{\varepsilon}=\varepsilon_{0}+\frac{4 \pi \lambda \omega_{0}^{2}}{\omega_{0}^{2}-\omega^{2}+i \omega \tau}
$$


Here $\varepsilon_{0}$ is the background permittivity, $4 \pi \lambda$ is the oscillator force of transition into electron state with resonant frequency $\omega_{0}, \tau$ is the damping factor.

According to Eq. (1), the energy coefficient of reflection $R$ and transmission $T$, and the tangent of phases $\tan \phi$ and $\tan \Phi$ of light are defined as follows

$R=\frac{\sigma_{12}^{2}+\Theta^{2}+2 \sigma_{12} \Theta \cos F_{-}}{1+\sigma_{12}^{2} \Theta^{2}+2 \sigma_{12} \Theta \cos F_{+}}$,

$T=\frac{n_{3}}{n_{1}} \frac{T_{12} T_{23} \Omega}{1+\sigma_{12}^{2} \Theta^{2}+2 \sigma_{12} \Theta \cos F_{+}}$,

and

$$
\begin{aligned}
& \tan \phi=\frac{\operatorname{Im} \tilde{r}}{\operatorname{Re} \tilde{r}}= \\
& =\frac{\sigma_{12}\left(1-\Theta^{2}\right) \sin \phi_{12}+\Theta\left(1-\sigma_{12}^{2}\right) \sin \left(\phi_{23}-\operatorname{Re} \tilde{\delta}\right)}{\sigma_{12}\left(1+\Theta^{2}\right) \cos \phi_{12}+\Theta\left(1+\sigma_{12}^{2}\right) \cos \left(\phi_{23}-\operatorname{Re} \tilde{\delta}\right)},
\end{aligned}
$$

$\tan \Phi=\frac{\operatorname{Im} \tilde{t}}{\operatorname{Re} \tilde{t}}=$

$$
=-\frac{\sin \left(\frac{1}{2} \operatorname{Re} \tilde{\delta}\right)+\sigma_{12} \Theta \sin \left(F_{+}-\frac{1}{2} \operatorname{Re} \tilde{\delta}\right)}{\cos \left(\frac{1}{2} \operatorname{Re} \tilde{\delta}\right)+\sigma_{12} \Theta \cos \left(F_{+}-\frac{1}{2} \operatorname{Re} \tilde{\delta}\right)}
$$

where $\quad \Omega=\exp (-\operatorname{Im} \tilde{\delta}), \quad T_{12,23}=\tilde{t}_{12,23} \cdot \tilde{t}_{12,23}^{*}$, $\tilde{r}_{12,23}=\sigma_{12,23} \exp \left(i \phi_{12,23}\right), F_{ \pm}=\phi_{12} \pm\left(\phi_{23}-\operatorname{Re} \tilde{\delta}\right)$.

Then expression for energetic coefficients of reflection and transmission are defined as [21, 24-26]:

$$
\begin{aligned}
& R=\frac{R_{\min }+b^{2} \cos ^{2} \frac{F_{-}}{2}}{1+b^{2} \cos ^{2} \frac{F_{+}}{2}}=\frac{R_{\max }-a^{2} \sin ^{2} \frac{F_{-}}{2}}{1-a^{2} \sin ^{2} \frac{F_{+}}{2}} \text { and } \\
& T=\frac{T_{\min }}{1-a^{2} \sin ^{2} \frac{F_{+}}{2}}=\frac{T_{\max }}{1+b^{2} \cos ^{2} \frac{F_{+}}{2}},
\end{aligned}
$$

where $\quad a^{2}=\frac{4 \sigma_{12} \Theta}{\left(1+\sigma_{12} \Theta\right)^{2}} \quad$ and $\quad b^{2}=\frac{4 \sigma_{12} \Theta}{\left(1-\sigma_{12} \Theta\right)^{2}}$, $\Theta=\sigma_{23} \Omega$. The functions

$R_{\text {max } \text { min }}=\left(\frac{\sigma_{12} \pm \Theta}{1 \pm \sigma_{12} \Theta}\right)^{2}$ and $T_{\text {max, min }}=\frac{n_{3}}{n_{1}} \frac{T_{12} T_{23} \Omega}{\left(1 \mp \sigma_{12} \Theta\right)^{2}}$

are the envelope functions of Fabry-Perot spectra.

\section{Discussion}

1. SLSFP with resonant dispersion (2). In many practical problems, medium with refraction $n_{1}$ is air or vacuum and $n_{1}=1$. For a free layer with resonant dispersion of $\widetilde{\varepsilon}(\omega)$, the calculated spectra $R(\omega), T(\omega)$ $\phi(\omega), \quad \Phi(\omega)$ and their envelope functions $(R, T, \phi)_{\max , \min }$ are shown in Fig. 2. In the region of resonant absorption near the resonant frequency $\omega_{0}$, it is possible to separate a frequency interval with width $\Delta \omega_{p}$, which is bounded by an interval with significant absorption where $\Omega \rightarrow 0$. Inside the interval $R_{\max } \approx R_{\min }$, and the spectra are formed as if the light wave is reflected from a semi-infinite medium with resonant dispersion (2) [21, 24-26]. The distance between envelope functions $\Delta R=R_{\max }-R_{\min }$ and $\Delta T=T_{\max }-T_{\min }$ are equal to

$$
\begin{aligned}
& \Delta R=\frac{4 \sigma_{12} \Theta}{\left(1-\sigma_{12}^{2} \Theta^{2}\right)^{2}}\left(1-\sigma_{12}^{2}\right)\left(1-\Theta^{2}\right) \text { and } \\
& \Delta T=\frac{4 \sigma_{12} \Theta \Omega}{\left(1-\sigma_{12}^{2} \Theta^{2}\right)^{2}} \frac{n_{3}}{n_{1}} T_{12} T_{23} .
\end{aligned}
$$

The absorption level increases with approaching to the resonant frequency $\omega_{0}$, and the value $\Delta R \rightarrow 0$ because of damping factor approaching to null $\Omega \rightarrow 0$. Near the resonant region beyond the interval $\Delta \omega_{p}$, the functions (Fig. 1b)

$\phi_{\max , \min } \cong 2 \pi \pm \frac{\sigma_{12}\left(1-\sigma_{23} \Theta\right) \sin \phi_{12}+\sigma_{23}\left(1-\sigma_{12}^{2}\right) \Omega}{\sigma_{12}(1+\Theta) \cos \phi_{12}}$

are the envelope functions of the phase spectrum of reflection. It is problematic to apply the envelope function method (Fig. 1b) to describe the phase spectrum $\Phi(\omega)$ for light transmitted through the layer.

At the arbitrary frequency, we have the following expression:

$\frac{R_{\max }-R}{R-R_{\min }}=\frac{T-T_{\min }}{T_{\max }-T}=\left(\frac{a}{b}\right)^{2} \tan ^{2} \frac{F_{ \pm}}{2}$.

The ratios $\frac{R_{\max }-R}{R-R_{\min }}$ and $\frac{T_{\max }-T}{T-T_{\min }}$ vary from 0 to $+\infty$, and at the frequencies $\omega_{1,2}$ from both side of extreme contour peak the ratios are equal to 1 . Hence, coefficients for reflected and transmitted light at the frequencies $\omega_{1,2}$ equal (Fig. 2)

$\Sigma R=\frac{1}{2}\left[R_{\text {max }}+R_{\text {min }}\right]$ and $\Sigma T=\frac{1}{2}\left[T_{\text {max }}+T_{\text {min }}\right]$.

It is the approach (11) that enables to ground the validity of interferogram apparatus characteristic determination $[16,17]$. The extreme contour width $\Delta \omega$ under the condition (11) equals $\Delta \omega=\frac{c_{0}}{2 n_{2} d} \Delta \delta$, where $\Delta \delta$ is the width contour extreme in phase units (phase distance between frequencies $\omega_{1,2}$ on both sides from its peak). 


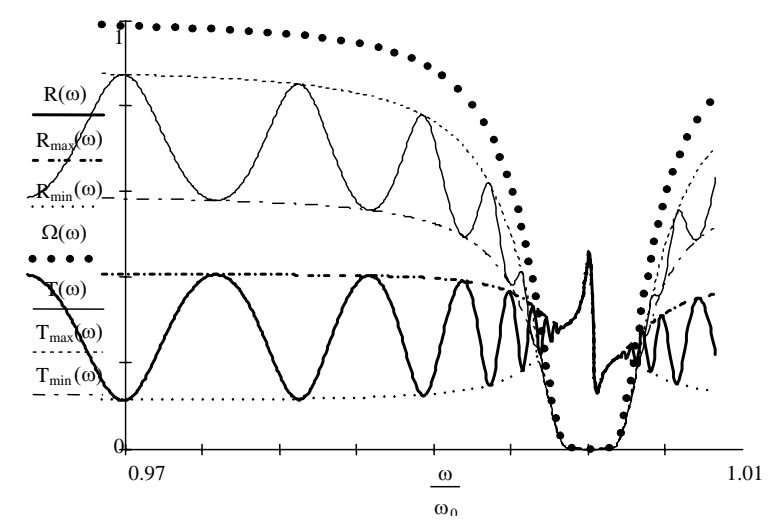

a)

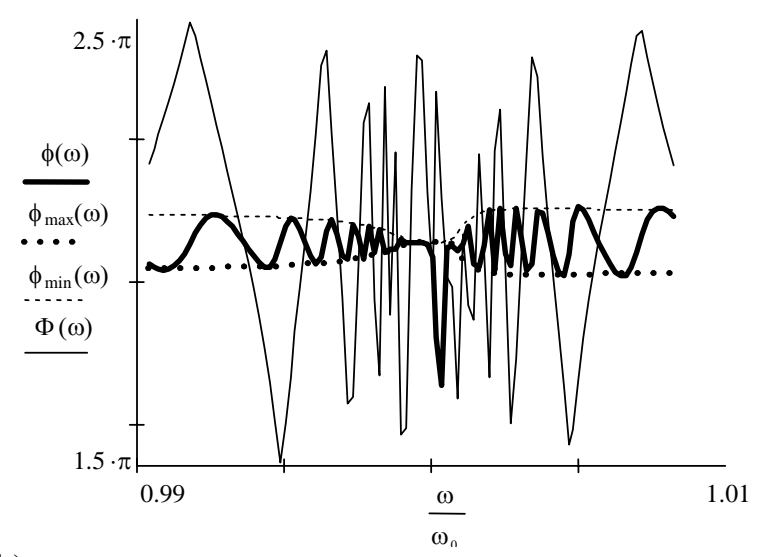

b)

Fig. 1. Calculated spectra of $R(\omega), T(\omega)$ in the resonant region and their envelope functions $(R, T)_{\max , \min }$ (a) and $\phi(\omega)$, $\Phi(\omega)(\mathrm{b})$.

The phase width $\Delta \delta$ equals $\Delta \delta=2 \pi \frac{\Delta \omega}{\Delta \omega_{\text {min }}}$, where $\Delta \omega_{\min }$ is the distance between the frequencies on both sides from the maximum.

The $2 \pi$-periodicity of Fabry-Perot spectra allows to determine the area restricted by the contour of a maximum minus the area restricted by envelope function of adjacent minima through the structure parameters as

$S_{R}=2 \int_{\pi / 2}^{3 \pi / 2}\left[R(\zeta)-R_{\min }\right] d \zeta$

where $\zeta=\frac{\operatorname{Re} \tilde{\delta}}{2}$. For transparent structures the area $S_{R}$ is equal

$$
S_{R}=\left(1-R_{\min }\right)\left[\pi-\int_{\pi / 2}^{3 \pi / 2} \frac{d \zeta}{1+b^{2} \cos ^{2} \zeta}\right] .
$$

This integral is the standard integral [28].

In the region of absorption the integration procedure can be simplified by replacement $R_{\min }$ at the maximum frequency by the mean value of two adjacent

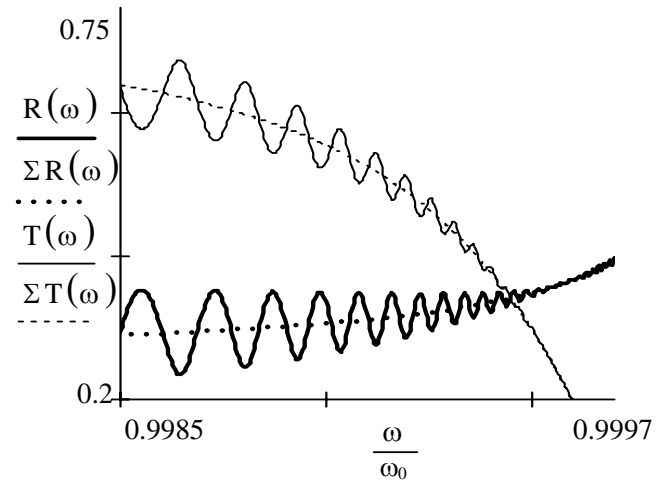

Fig. 2. Calculated spectra of $R(\omega), T(\omega)$ in the resonant region and contours $\Sigma R(\omega)$ and $\Sigma T(\omega)$.

minima $\frac{1}{2}\left(R_{\min , m-1}+R_{\min , m+1}\right)$ [22]. Clearly, this way can be applied to the transmitted waves.

2. Free layer and the fixed one on substrate surfaces with constant absorption $\chi_{2}=$ const . The values of energetic coefficients $(R, T)_{\max , \min }$ in the absorptive films are a function of $\lambda$. Even for absorptive layers with constant absorption, energetic coefficients $(R, T)_{\max , \min }$ vary with variation of $\lambda$. According to the definition given by Michelson the visibility of the interferogram $V=\frac{R_{\text {max }}-R_{\text {min }}}{R_{\text {max }}+R_{\text {min }}}$. Then, according to the expression (7) we have

$V=2\left[\frac{\sigma_{12}\left(1-\Theta^{2}\right)}{\left(1-\sigma_{12}{ }^{2}\right) \Theta}+\frac{\left(1-\sigma_{12}^{2}\right) \Theta}{\sigma_{12}\left(1-\Theta^{2}\right)}\right]^{-1}$.

Using the transformation

$2 \frac{x+y}{x-y}=\frac{\sqrt{x}+\sqrt{y}}{\sqrt{x}-\sqrt{y}}+\frac{\sqrt{x}-\sqrt{y}}{\sqrt{x}+\sqrt{y}}$,

we obtained that $V=2\left[W^{-1}+W\right]^{-1}$, where $W=$ $=\frac{\sqrt{R_{\max }}-\sqrt{R_{\min }}}{\sqrt{R_{\max }}+\sqrt{R_{\min }}}=\frac{\Theta}{\sigma_{12}} \frac{1-\sigma_{12}^{2}}{1-\Theta^{2}}$. Therefore, when the absorption index $\chi_{2}$ does not depend on frequency, the relative slope between linear dependences $\ln W^{-1}$ and $\operatorname{Im} \tilde{\delta}$ does not depend on frequency for an arbitrary ratio between the refraction indices $n_{1,2,3}$ (Fig. 3) and the relation

$\ln W^{-1}=$ const $+\exp (\operatorname{Im} \tilde{\delta})$ 


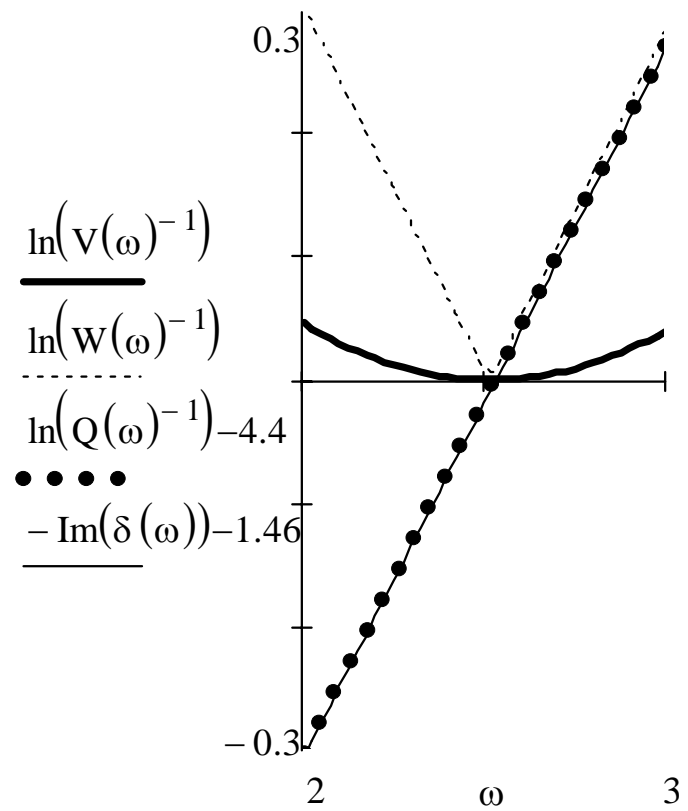

Fig. 3. Calculated dependences of $\ln W^{-1}, \ln V^{-1}$ and $\operatorname{Im} \tilde{\delta} \quad$ for parameters: $n_{1}=1, \quad n_{2}=1.25, \quad n_{3}=3.5$, $\chi_{2}=0.0038, d=15 \mu \mathrm{m}$.

is valid with accuracy to constant. Slope of $\ln V$ depends on the ratio between the refraction indices $n_{1,2,3}$.

\section{Principal conclusions}

Amplitude-phase Fabry-Perot spectra for single-layer structures at the normal can be described by the envelope functions $(R, T)_{\max , \min }$.

The width of the interference band as a phase separation between the points, for which the reflectance are $\frac{1}{2}\left[(R, T)_{\max }+(R, T)_{\min }\right]$, is determined through instrumental characteristics of the Fabry-Perot interferogram of single-layer structures.

In the region of constant light absorption in film, the slope of $\ln \left(\frac{\sqrt{R_{\max }}-\sqrt{R_{\min }}}{\sqrt{R_{\max }}+\sqrt{R_{\min }}}\right)$ equals to that of $\operatorname{Im} \tilde{\delta}$.

\section{References}

1. C. Fabry, A. Perot, Theori et applications d'une nouvelle methode de spectroscopie interfrentielle // Ann. Chim. Phys. Peris.16, p. 115-144 (1899).

2. M. Born and E. Wolf, Principles of optics. Pergamon, New York, 1970.
3. A. Thelen, Design of optical interference coatings. McGraw-Hill, New York, 1989.

4. A. Ezbiri, R. Tatam, Passive signal processing of miniature Fabry-Perot interferometric sensors // Proc. SPIE 2341 , p. 114-116 (1994).

5. A. Garcia-Valenzuela, M.C. Pena-Gomar, J. Villatoro, Sensitivity analysis of angle-sensitive detectors based on a film resonator // Opt. Eng. 42(4), p. 1084-1092 (2003).

6. R. Goldhanh, S. Shokhovets, J. Scheiner, et. al., Determination of group III nitride film properties by reflectance and spectroscopic ellipsometry studies // Phys. status solidi (a) 177, p. 107-115 (2000).

7. S.A. Kovalenko, M.P. Lisitsa // Zhurnal prikladnoi spektroskopii 69, p. 388-394 (2002) (in Russian).

8. L.A. Holovan', A.F. Konstatinova, K.B. Imahnazieva et. al., Anisotropy of optical dispersion in a layer of nanoporous silicon // Kristalografija 49(1), p. 151-156 (2004) (in Russian).

9. R. Swanepoel, Determination of thickness and optical constants of amorphous silicon // J. Phys. E: Sci. Instrum. 16, p. 1214-1222 (1983).

10. R. Swanepoel, Determination of surface roughess and optical constants of inhomogeneous amorphous silicon films // J. Phys. E: Sci. Instrum. 17, p. 896903 (1984).

11. D.B. Kushev, N.N. Zheleva, Y. Demakopoluou, D. Siapkas, A new method of the determination of the thickness, optical constants, and relaxation time of weakly absorbing semiconductor thin films // Infrared Phys. 26 (6), p. 385-393 (1986).

12. D. Siapkas, D.B. Kushev, N.N. Zheleva, J. Siapkas, I. Lelidis, Optical constants of tin-telluride determined from infrared interference spectra // Infrared Phys. 31(5), p. 425-433 (1991).

13. D.B. Kushev, N.N. Zheleva, Transmittivity, reflectivities and absorptivities of a semiconductor film with a linear variation in thickness // J. Phys. D: Appl. Phys. 8, p. 1239-1243 (1995).

14. D.B. Kushev, Influence of finite spectral width on the interference spectra of a thin absorbing film on a transparent substrate // Infrared Phys. and Technology 37, p. 259-264 (1996).

15. V.V. Fillipov, V.P. Kutjavichus, Accuracy of determination of the optical parameters of thin films by the method of the reflectance spectrum extrema envelopes // Zhurn. prikladnoy spektroskopii 70(1), p. 109-115 (2003) (in Russian).

16. P.S. Kosobutskyy, M.S. Karkulovska and M.S. Seheda, Physics basics for modeling the electromagnetic wave processes in optics. Lvivska Politekhnika Publ., Lviv, Ukraine (2003) (in Ukrainian).

17. P.S. Kosobutskyy, M.S. Karkulovska, Ya.P. Kosobutskyy, Bandwidth, sharpness, and visibility of resonances in light reflectance and transmittance of free monolayer Fabry-Perot interferometers // 
Optika i spektroskopiya 94(1), p. 71-74 (2003) (in Russian).

18. P.S. Kosobutskyy, M.S. Karkulovska, and Ya.P. Kosobutskyy, On phase-amplitude correlation in reflection spectra of Fabry-Perot interferometers // Optika i spektroskopiya 94(3), p. 434-436 (2003) (in Russian).

19. M.V. Lobur and Ya.P. Kosobutskyy // Akusticheskiy Zhurnal 50(4), p. 1-2 (2004) (in Russian).

20. P.S. Kosobutskyy, Inversion of a non-monotonic polarozational angular dependence of the light reflection coefficient from a thin film on an absorbing substrate // Zhurnal prikladnoy spektroskopii 72(2), p. 277-279 (2005) (in Belorussian).

21. P.S. Kosobutskyy, A. Morgulis, Simulation of onelayer Fabry-Perot interferometers by the method of enveloping spectra of reflection and transmission of light // Opticheskiy Zhurnal 71(12), p. 63-68 (2004) (in Russian).
22. P.S. Kosobutskyy, A. Morgulis // Zhurnal prikladnoy spektroskopii 71(5), p. 656-672 (2004) (in Belorussian).

23. P.S. Kosobutskyy, A. Morgulis, M.S. Karkulovska, and A.B.Danilov // Ukrainsky Zhurnal Fizich. Optyky 3(5), p. 100-103 (2004) (in Ukrainian).

24. P.S. Kosobutskyy, A. Morgulis // Ukrainsky Fizich. Zhurnal 49(12), p. 1163-1166 (2004) (in Ukrainian).

25. P.S.Kosobutskyy, A. Morgulis // Ukrainsky Fizichny Zhurnal 50(3), p. 230-234 (2005) (in Ukrainian).

26. P.S. Kosobutskyy, A. Morgulis, A.B. Danilov, and M.S. Karkulovska // Ukrainsky Fizich. Zhurnal 50(6), p. 551-555 (in Ukrainian).

27. J.J. Hopfield and D.G. Thomas, Theoretical and experimental effects of spatial dispersion on the optical properties of crystals // Phys.Rev. 132, p. 563-573 (1963).

28. H.B. Dwigt, Tables of integrals and other mathematical data. McMillan Company, New York, 1961. 